Objectives We aimed to compare clinical and survival outcomes in high grade ovarian cancer (HGOC) stratified by homologous recombination deficiency (HRD) status undergoing frontline and/or maintenance therapy.

Methods We performed a retrospective analysis of HGOC from April 2013 to June 2019. Clinical outcomes were analyzed by (1) germline BRCA+ (2) germline BRCA - and somatic BRCA/HRD+, or (3) BRCA-/HRD-. Progression free (PFS) and overall survival (OS) were estimated using KaplanMeier methods and modeled via Cox proportional hazards regression.

Results 187 patients met inclusion criteria. 106 patients had germline BRCA mutation, 26 somatic BRCA/HRD+, and 55 BRCA/HRD-. Multivariate analysis for PFS revealed that age (HR 1.02, 95\% CI 1.00-1.04), $\mathrm{p}=0.01$ ), stage (HR 5.7, 95\% CI 1.39-23.4, p=0.02), R0 resection at TRS (HR 0.41, 95\% CI $0.21-0.83, \mathrm{p}=0.01$ ), and BRCA/HRD- status (HR 1.63, $95 \%$ CI 1.07-2.48, $\mathrm{p}=0.02$ ) were significant factors impacting PFS. Multivariate analysis for OS revealed age (HR 1.07, 95\% CI 1.03-1.10, $\mathrm{p}<0.001$ ) and R0 resection at TRS (HR 0.19, 95\% CI $0.08-0.44, \mathrm{p}<0.001)$ were significant factors impacting OS. 17 of 187 patients received PARPi maintenance therapy. All harbored a germline or somatic mutation in BRCA1/ BRCA2 (14) or had tumors characterized by HRD (3). Multivariate analysis for PFS revealed that PARPi maintenance therapy (HR 0.14 95\% CI 0.04-0.57), p=0.006) was a significant factor impacting PFS.

Conclusions Germline BRCA-mutant, somatic BRCA/HRD+ HGOC was associated with improved PFS and OS regardless of primary TRS or NACT. BRCA-/HRD- was a negative prognostic factor for survival in HGOC. PARPi maintenance therapy was associated with improved PFS in Germline BRCAmutant, somatic BRCA/HRD+ HGOC

\section{OP024/\#182 FINDINGS AND OUTCOMES IN A POST- VACCINATION COHORT OF YOUNG WOMEN UNDER 25 YEARS ATTENDING A TERTIARY COLPOSCOPY SERVICE}

${ }^{1} \mathrm{C}$ Yim*, ${ }^{2} Y$ Jayasinghe, ${ }^{2} \mathrm{D}$ Wrede, ${ }^{2} \mathrm{~J}$ Tan. ${ }^{1}$ Royal Women's Hospital, Gynaecologic Cancer Centre, Randwick, Australia; ${ }^{2}$ Royal Women's Hospital, Dysplasia, Parkville, Australia

10.1136/ijgc-2021-IGCS.41

Objectives In 2007, human papillomavirus (HPV) vaccination was rolled out in Australia, with a high uptake of $73 \%$, and a consequent reduction in high-grade dysplasia in young women. The aim was to provide descriptive data on post-vaccination women below 25 years between 2008 and 2017, prior to the change in cervical screening guidelines.

Methods A retrospective cohort analysis of women under 25 attending a tertiary colposcopy clinic.

Results 3128 women with a median age of 22 (range 14-24) years were identified. When comparing overall worst histology result, vaccinated women were less likely to have a high grade abnormality than unvaccinated women (RR 0.78, 95\%CI $0.67-0.90, p=0.0006)$. Amongst those with high grade abnormalities, there was no significant difference in rates of CIN2 or CIN3 between vaccinated and unvaccinated women (RR 0.81, 95\%CI 0.62-1.05, $\mathrm{p}=0.1086$ ).

Conclusions This study provides baseline data on young women under the previous cervical screening program, following the introduction of the HPV vaccine.

\section{OP025/\#128 COMPREHENSIVE PERIOPERATIVE CARE PROGRAM TO IMPROVE SAME-DAY DISCHARGE AFTER MINIMALLY INVASIVE GYNECOLOGIC ONCOLOGY SURGERY}

${ }^{1} \mathrm{RS}$ Kim, 'S Laframboise, ${ }^{2} \mathrm{G}$ Nelson, ${ }^{3} \mathrm{~S}$ Mccluskey, ${ }^{4} \mathrm{~L}$ Avery, ${ }^{1} \mathrm{~N}$ Kujbid, ${ }^{1} \mathrm{~A}$ Zia, ${ }^{1} \mathrm{M}$ Bernardini, ${ }^{1} \mathrm{~S}$ Ferguson, ${ }^{1} \mathrm{~T}$ May, ${ }^{1} \mathrm{~L}$ Hogen, ${ }^{1} \mathrm{P}$ Cybulska, ${ }^{1} \mathrm{G}$ Bouchard-Fortier. ${ }^{1}$ Princess Margaret Cancer Centre/University of Health Network/Sinai Health Systems, Gynecologic Oncology, Toronto, Canada; ' $C$ Cumming School of Medicine, Obstetrics and Gynecology, Calgary, Canada; ${ }^{3}$ Toronto General Hospital, University Health Network, Anesthesia and Pain Management, Toronto, Canada; ${ }^{4}$ Princess Margaret Cancer Centre, Biostatistics, Toronto, Canada

\subsection{6/ijgc-2021-IGCS.42}

Objectives Same-day discharge (SDD) after minimally invasive hysterectomy for gynecologic conditions has been shown to be safe and feasible. We designed and implemented a quality improvement perioperative program to improve SDD rate from $30 \%$ to $75 \%$ over a 12 -month period.

Methods We included 102 consecutive patients undergoing minimally invasive hysterectomy at a single cancer centre during the 12-month implementation period. A pre-intervention cohort of 100 patients was identified for comparison of clinicodemographic variables and perioperative outcomes. We developed a comprehensive perioperative care program based on Early Recovery after Surgery (ERAS) principles and met biweekly for plan-do-study-act (PDSA) cycles. Patients were followed for 30 days after discharge. We used a run chart to monitor the effects of our interventions and conducted a multivariate analysis to determine patient factors or interventions associated with SDD.

Results SDD rate increased from $29 \%$ to $75 \%$ after implementation $(p<0.001)$. The post implementation cohort was significantly younger $(59$ vs. $65 y r s ; p=0.025)$ and had shorter operative times $(180$ vs. 211 minutes; $\mathrm{p}<0.001)$ but the two groups were similar in BMI, comorbidity, stage, and intraoperative complications. There was no difference in 30-day perioperative complications, readmissions, reoperations, emergency department visits, or mortality. The most common reason for overnight admission post intervention was nausea and vomiting (16\%). Overall, $89 \%$ of patients rated their experience as 'very good' or 'excellent', and $87 \%$ felt that their post-operative length of stay was adequate.

Conclusions Following implementation of a perioperative quality improvement program, our interventions significantly improved SDD rates while maintaining low 30-day perioperative complications and excellent patient experience.

\section{OP026/\#45 MALNUTRITION AS A RISK FACTOR FOR POST- OPERATIVE MORBIDITY IN GYNECOLOGIC CANCER: ANALYSIS USING THE NATIONAL SURGICAL QUALITY IMPROVEMENT PROGRAM (NSQIP) DATABASE}

${ }^{1} E$ Goins*, ${ }^{2} \mathrm{~J}$ Weber, ${ }^{2} \mathrm{~T}$ Truong, ${ }^{3} \mathrm{H}$ Moss, ${ }^{3} \mathrm{R}$ Previs, ${ }^{3} \mathrm{~B}$ Davidson, ${ }^{3} \mathrm{~L}$ Havrilesky. ${ }^{1}$ Duke University, School of Medicine, Durham, USA; ${ }^{2}$ Duke University Health System, Duke Clinical Research Institute, Durham, USA; ${ }^{3}$ Duke University School of Medicine, Obstetrics and Gynecology, Durham, USA

\subsection{6/ijgc-2021-IGCS.43}

Objectives Malnutrition increases risk of post-surgical morbidity in gynecologic malignancies. We assessed whether different malnutrition definitions are suitable for predicting morbidity in each cancer type. 
Methods Patients undergoing resection of ovarian, uterine, or cervical cancer between 2005-2019 were identified using the NSQIP database. Body mass index (BMI), weight loss, and albumin were used to evaluate whether patients met various malnutrition criteria (severe, ESPEN1, ESPEN2, ACS, mild, albumin $<3.5 \mathrm{~g} / \mathrm{dL}$; figure 1 ). Outcomes included 30-day major post-operative complications, readmission, and reoperation. Modified Poisson regression was used to estimate the association between each definition and outcomes using risk ratios (RR) and 95\% confidence intervals (CI).

Results Ovarian cancer patients meeting ESPEN2 had higher risk of readmission ( $R R$ 1.69;1.29-2.20), reoperation (RR $2.53 ; 1.70-3.77$ ), and complications (RR 1.36; 1.20-1.54; Table). Uterine cancer patients meeting ACS had increased risk of readmission ( $R R$ 2.74;2.09-3.59), reoperation ( $R R$ $3.61 ; 2.29-5.71$ ) and complications (RR 3.92;3.40-4.53). For cervical cancer, albumin $<3.5$ was associated with readmission (RR 1.48;1.01-2.19), reoperation (RR 2.25;1.17-4.34), and complications (RR 2.59;2.11-3.17). Albumin $<3.5$ was also associated with increased risk of all outcomes for ovarian and uterine cancer patients.

Conclusions The malnutrition definitions predicting the highest number of adverse post-operative outcomes varies by cancer type. Major complications, readmission, and reoperation were

\begin{tabular}{|c|c|c|c|c|c|}
\hline $\begin{array}{c}\text { Severe } \\
\text { Malnutrition } \\
\text { BMI }<18.5+ \\
\text { weight loss }{ }^{*}\end{array}$ & $\begin{array}{c}\text { ESPEN } 1 \\
\text { If }<70 \text { yo: BMI 18.5- } \\
20+\text { weight loss* } \\
\text { OR } \\
\text { If } 270 \text { yo: BMI 18.5- } \\
22+\text { weight loss* }\end{array}$ & $\begin{array}{c}\text { ESPEN } 2 \\
\text { BMI }<18.5\end{array}$ & $\begin{array}{c}\text { ACS } \\
\text { If }<70 \text { yo: BMI } \\
>20+\text { weight } \\
\text { loss* } \\
\text { OR } \\
\text { If } \geq 70 \text { yo: BMI } \\
>22+\text { weight } \\
\text { loss* }\end{array}$ & $\begin{array}{c}\text { Mild } \\
\text { Malnutrition } \\
\text { If }<70 \text { yo: } B M \mid \\
18.5-20 \\
O R \\
\text { If } \geq 70 \text { yo: } B M \mid \\
18.5-22\end{array}$ & $\begin{array}{l}\text { Albumin } \\
<3.5 \mathrm{~g} / \mathrm{dL}\end{array}$ \\
\hline
\end{tabular}

Abstract OP026/\#45 Figure 1 Malnutrition definitions

Abstract OP026/\#45 Table 1 Association between malnutrition definitions and outcones by cancer presented as risk ratios and $95 \%$ confidence intervals

\begin{tabular}{|c|c|c|c|}
\hline & Uterine & Cervical & Ovarian \\
\hline \multicolumn{4}{|c|}{ Major complication(s) $^{1}$} \\
\hline Mild malnutrition & $1.33(1.14,1.55)$ & $1.69(1.30,2.18)$ & $1.16(1.07,1.26)$ \\
\hline Severe malnutrition & $7.85(6.10,10.11)$ & $4.83(2.54,9.21)$ & $2.05(1.66,2.53)$ \\
\hline ESPEN 1 & $3.65(2.23,5.97)$ & $3.62(1.53,8.54)$ & $1.65(1.34,2.01)$ \\
\hline ESPEN 2 & $1.74(1.36,2.22)$ & $1.92(1.32,2.79)$ & $1.36(1.20,1.54)$ \\
\hline ACS & $3.92(3.40,4.53)$ & $3.51(2.49,4.95)$ & $1.67(1.52,1.82)$ \\
\hline Albumin $<3.5 \mathrm{~g} / \mathrm{dL}^{3}$ & $3.74(3.48,4.02)$ & $2.59(2.11,3.17)$ & $1.74(1.65,1.83)$ \\
\hline \multicolumn{4}{|c|}{ Unplanned readmission $^{2}$} \\
\hline Mild malnutrition & $1.17(0.93,1.48)$ & $1.46(1.00,2.15)$ & $0.96(0.78,1.18)$ \\
\hline Severe malnutrition & $\cdots$ & - & $\cdots$ \\
\hline ESPEN 1 & - & - & $\ldots$ \\
\hline ESPEN 2 & $1.15(0.74,1.79)$ & $1.33(0.71,2.50)$ & $1.69(1.29,2.20)$ \\
\hline ACS & $2.74(2.09,3.59)$ & $2.45(1.24,4.82)$ & $1.36(1.06,1.75)$ \\
\hline Albumin $<3.5 \mathrm{~g} / \mathrm{dL}^{3}$ & $2.38(2.10,2.69)$ & $1.48(1.01,2.19)$ & $1.28(1.11,1.47)$ \\
\hline \multicolumn{4}{|c|}{ Unplanned reoperation $^{2}$} \\
\hline Mild malnutrition & $0.98(0.61,1.58)$ & $0.91(0.38,2.21)$ & $0.95(0.66,1.38)$ \\
\hline Severe malnutrition & - & - & - \\
\hline ESPEN 1 & - & - & - \\
\hline ESPEN 2 & $1.25(0.56,2.77)$ & $1.42(0.46,4.38)$ & $2.53(1.70,3.77)$ \\
\hline ACS & $3.61(2.29,5.71)$ & $2.23(0.57,8.69)$ & $1.09(0.65,1.81)$ \\
\hline Albumin $<3.5 \mathrm{~g} / \mathrm{dL}^{3}$ & $2.56(2.01,3.25)$ & $2.25(1.17,4.34)$ & $1.31(1.01,1.70)$ \\
\hline \multicolumn{4}{|c|}{$\begin{array}{l}{ }^{1} \text { Major complications included unplanned intubation, ventilator use }>48 \text { hours, sepsis, septic shock, } \\
\text { pneumonia, deep incisional surgical site infection, acute renal failure, organ space surgical site } \\
\text { infection, renal insufficiency, wound disruption, pulmonary embolism, myocardial infarction, cardiac } \\
\text { arrest requiring CPR, stroke/cerebrovascular accident with neurological deficit, deep vein thrombosis, } \\
\text { blood transfusion } \\
{ }^{2} \text { Outcome only available starting in } 2011 \\
{ }^{3} \text { Only among the subset of patients with pre-operative serum albumin } \\
\ldots-=\text { models did not converge due to low event rate after dividing by malnutrition definition and cancer } \\
\text { type }\end{array}$} \\
\hline
\end{tabular}

associated with $\mathrm{BMI}<18.5$ alone for ovarian cancer (ESPEN2), with $10 \%$ recent weight loss and a normal or overweight BMI for uterine cancer (ACS), and with albumin $<3.5$ for all cancers. These criteria may be useful for cancer-specific preoperative planning.

\section{OP027/\#469 ELECTRONIC PATIENT-REPORTED OUTCOME (EPRO) MEASURES IN GYNECOLOGIC ONCOLOGY: INITIAL EXPERIENCE AFTER WORKFLOW IMPLEMENTATION}

J Noh*, C-H Choi, T-J Kim, J-W Lee, Y-Y Lee. Samsung Medical Center, Obstetrics and Gynecology, Seoul, Korea, Republic of

\subsection{6/ijgc-2021-IGCS.44}

Objectives The aim of this study was to report our initial experience with a mobile app of electronic patient-reported outcome (ePRO) for patients undergoing treatment for gynecologic malignancies.

Methods The target patients were introduced to a mobile app in which they could answer to pre-selected questions. The questions included the quantification of fatigue, pain, anxiety, dizziness, hair loss, peripheral numbness, tingling, nausea, myalgia, depression, insomnia and others. Two different sets of questions were used for surgery and chemotherapy.

Results A total of 61 patients reported more than 29,000 data points. The mean ages were $53.0 \pm 12.2$ years old for the surgery group and $54 \pm 13.2$ years old for the chemotherapy group. The median numbers of app use during the course of treatment was 10 and 13 for the surgery and chemotherapy groups, respectively. The mean duration of app use to complete each report was $8 \pm 13$ minutes for the surgery and 7 \pm 12 minutes for the chemotherapy groups. This did not differ by age groups, suggesting that there were no difficulties of using the app for any specific age group. ePRO was able to detect the occurrence of both expected and unexpected side effects. In addition, a gradual increase in the severity of side effects over the course of treatment, especially for those who received chemotherapy, could be observed.

Conclusions ePRO have a great potential to improve patient care in gynecologic oncology by providing a comprehensive documentation of symptoms and side effects.

\section{Surgical Film Abstracts}

\section{SF001/\#65 LAPAROENDOSCOPIC RADICAL TRACHELECTOMY AND PELVIC LYMPHADENECTOMY WITHOUT UTERINE MANIPULATOR}

H Lu*, C Huo, Z Lin. The Sun Yat-Sen memorial hospital of Sun Yat-Sen University, Gynecologic Oncology, Guangzhou, China

\subsection{6/ijgc-2021-IGCS.45}

Introduction In face of the postponement in marital and reproductive age in the modern society, most of the patients with cervical cancer have not conceived yet or still bear fertility plans. We herein introduce the Laparoendoscopic radical trachelectomy (LRT) a surgery called radical trachelectomy through a minimally invasive approach for young patients with cervical cancer to preserve fertility without compromising the oncology outcomes. 